\title{
Proton pump inhibitors: balancing the benefits and potential fracture risks
}

\section{J. Brent Richards MD, David Goltzman MD}

$\infty$ See related research paper by Targownik and colleagues, page 319

$\mathrm{P}$ roton pump inhibitors have been a cornerstone in the treatment of gastresophageal reflux disease and peptic ulcer disease since their introduction in the late 1980s. Gastresophageal reflux disease is a common disorder, and 12.4 million prescriptions for proton pump inhibitors were dispensed in Canada in 2004. ${ }^{1}$ It would therefore be of considerable public health importance if these medications substantially influenced a common disease. In this issue of $C M A J$, evidence is presented ${ }^{2}$ that supports and extends data from 2 other large administrative database studies, ${ }^{3,4}$ suggesting that the use of proton pump inhibitors is associated with increased risk of an osteoporotic fracture.

Using administrative data, Targownik and colleagues report that use of proton pump inhibitors was associated with increased risk of an osteoporosis-related fracture (vertebral, wrist or hip fracture) (odds ratio [OR] 1.92, 95\% confidence interval $[\mathrm{CI}] 1.16-3.18, p=0.04)$. In particular, there was a significant increase in the risk of hip fracture after 7 years of exposure to proton pump inhibitors (OR 4.55, 95\% CI $1.68-12.29, p=0.002)$.

Proton pump inhibitors, which are the most potent acidsuppressing drugs available, target the $\mathrm{H}^{+} / \mathrm{K}^{+}$ATPase of the gastric parietal cell and inhibit the final step in gastric proton release. Stomach acid and the slightly acidic milieu of the proximal duodenum appear necessary to liberate ingested calcium from its food matrix for absorption. ${ }^{5}$ Consequently, calcium malabsorption, negative calcium balance and potential bone loss may occur if acid production is suppressed. Nevertheless, it is unclear whether any calcium malabsorption is sufficiently severe to influence bone remodelling, and longterm studies on calcium malabsorption or the negative effects on skeletal metabolism are lacking. Furthermore, it is uncertain whether other mechanisms for skeletal weakening are active. Thus, the biologic plausibility of the relation between proton pump inhibitors and fracture, although compelling, requires further proof.

Observational studies that assess risks associated with therapeutic interventions are particularly prone to confounding because use of a medication is often associated with comorbidities, lifestyle behaviours or use of other medication that may also be associated with the event of interest. For example, a patient may be taking a proton pump inhibitor because of concomitant use of a nonsteroidal anti-inflammatory drug for a rheumatic disorder, both of which may increase the

\section{Key points}

- Three large studies using administrative databases have implicated the use of proton pump inhibitors as a risk factor for hip fractures.

- The relation between the use of proton pump inhibitors and fracture may involve calcium malabsorption due to induced hypochlorhydria, but this remains unproven.

- These retrospective administrative database studies should be confirmed by randomized controlled trials or prospective cohort studies.

- Clinicians must weigh the proven benefits of proton pump inhibitors when appropriately prescribed against the potential risk of osteoporotic fracture.

risk of fracture. Furthermore, administrative database studies are often unable to measure individual-level variables, such as use of over-the-counter medications and anthropomorphic measures, such as body mass index and bone mineral density, which both influence fracture risk. Therefore, residual confounding due to improperly measured or unmeasured variables may influence the reported associations.

However, statistical methods may be used to reduce this potential confounding by properly measuring and controlling for their influence in the study design and analysis. In their well-designed and executed study, Targownik and colleagues have appropriately used several of these methods, such as case-control matching and conditional logistic regression, to decrease the probability that their results are spurious.

Because of the above limitations, it is prudent to assess whether such an association between proton pump inhibitors and adverse events has been consistently described in other cohorts. Two previously published studies that used large administrative database also found an important relation between the use of proton pump inhibitors and osteoporotic fractures. In a study that included over 13000 cases with hip fractures and 135000 controls, Yang and colleagues ${ }^{3}$ found that proton pump inhibitor use was associated with increased risk of fracture (OR 1.44, 95\% CI 1.3-1.59) after just 1 year of therapy. This relation increased with higher doses and

From the Department of Medicine (Richards, Goltzman), McGill University, and the McGill University Health Centre (Goltzman), Montréal, Que.; and the Faculty of Medicine (Richards), King's College London, London, United Kingdom. 
longer durations. ${ }^{3}$ This study also found that histamine-2receptor antagonists were associated with increased risk of fracture, although to a lesser degree (OR 1.23, 95\% CI 1.14-1.39). Whether this suggests that both proton pump inhibitors and histamine-2-receptor antagonists act through a similar mechanism (i.e., calcium malabsorption due to induced hypochlorhydria) or are similarly confounded by their indication for therapy is currently unclear.

Intriguingly, in the study by Targownik and colleagues, a relation between the use of proton pump inhibitors and hip fractures was only observed after 5 years of therapy. This is not surprising, given that the skeletal effects of proton pump inhibitors may require years of exposure to the drug. Nevertheless, Yang and colleagues found such a relation after only 1 year of treatment. ${ }^{3}$ It is possible that this discrepancy is simply a matter of statistical power; the study by Yang and colleagues contained several-fold more patients with hip fractures than the study by Targownik and colleagues. This issue does, however, have considerable clinical significance and needs to be resolved.

Should these findings change clinical practice? Use of proton pump inhibitors is common, and given the efficacy of proton pump inhibitors, patients and their physicians are often reluctant to discontinue their use. Certainly, at one extreme, such as in patients with bleeding ulcers, the beneficial effects may far outweigh the risks associated with fracture. At the other extreme, liberal prescription of proton pump inhibitors for nebulous disorders for extended periods of time is likely worth revisiting. Nevertheless, further investigation into the issue of fracture and proton pump inhibitor use is warranted to provide evidence-based guidance for non-life-threatening cases in which proton pump inhibitors are appropriately prescribed over the long term.

The fact that 3 large, well-designed studies consistently report an association between the use of proton pump inhibitors and fractures and that this association appears to be both dose- and duration-dependent is certainly a strong basis for encouraging further investigation. Randomized controlled trials designed to assess the efficacy of acid-lowering medica- tions, but that also explicitly assess the incidence of osteoporotic fractures, would be the preferred experimental approach. However, it is likely that without these studies, physicians will remain reliant upon epidemiologic studies to examine the relation between proton pump inhibitors and fractures. Evidence from prospective cohort studies, which capture particular confounders unavailable to administrative database studies, may also be helpful.

Of course, clinicians and their patients are regularly burdened with the results of studies suggesting deleterious effects of medications that are used frequently in clinical practice. Physicians, together with their patients, must therefore always weigh the risks against the benefits of any particular medication. In the case of patients who use proton pump inhibitors, this equilibrium should be reconsidered with knowledge of the mounting, yet incomplete, evidence suggesting that proton pump inhibitors increase the risk of fracture.

Competing interests: None declared.

Contributors: Both of the authors contributed to the content of the article, revised it critically and approved the final version submitted for publication.

\section{REFERENCES}

1. Canadian Agency for Drugs and Technologies in Health. Current topics: proton pump inhibitors. Ottawa: The Agency; 2008. Available: www.cadth.ca/index .php/en/compus/current-topics/ppis (accessed 2008 May 27).

2. Targownik LE, Lix LM, Metge CJ, et al. Use of proton pump inhibitors and risk of osteoporosis-related fractures. CMAJ 2008;179:319-26.

3. Yang YX, Lewis JD, Epstein S, et al. Long-term proton pump inhibitor therapy and risk of hip fracture. JAMA 2006;296:2947-53.

4. Vestergaard P, Rejnmark L, Mosekilde L. Proton pump inhibitors, histamine H2 receptor antagonists, and other antacid medications and the risk of fracture. Calcif Tissue Int 2006;79:76-83.

5. Wright M J, Proctor DD, Insogna KL, et al. Protonpump-inhibiting drugs, calcium homeostasis, and bone health. Nutr Rev 2008;66:103-8.

Correspondence to: Dr. David Goltzman, Royal Victoria Hospital, 687 Pine Ave. W, Rm. H467 Montréal QC H3A 1A1;

fax 514 843-1712; david.goltzman@mcgill.ca

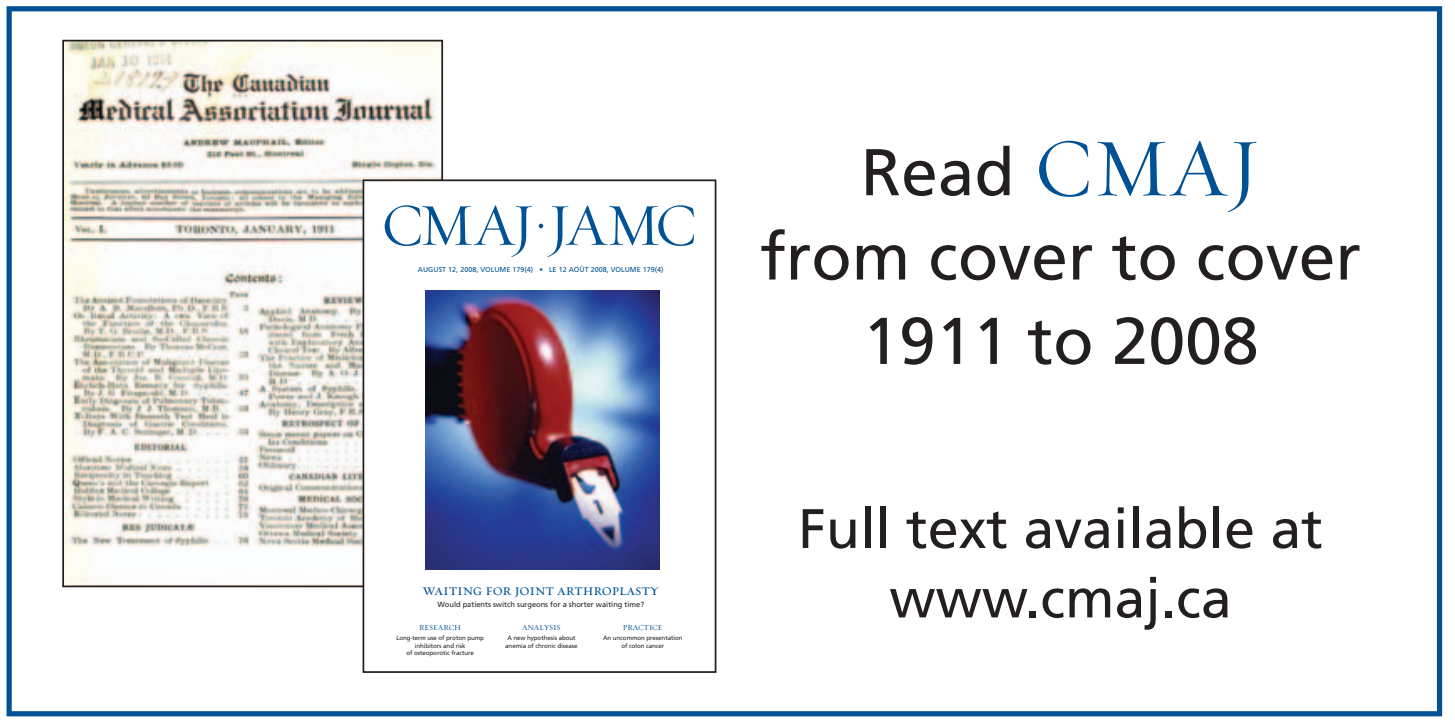

